REVISTA DE LA CEPAL 85 ABRIL 2005

\title{
El péndulo monetario en México
}

\author{
D avid I barra
}

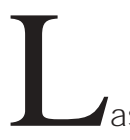

as visiones del Primer Mundo y la necesidad de hacer posible la convivencia entre naciones se decantan en un conjunto de reglas que forman el orden económico internacional de cada época. Esos órdenes son cambiantes, e implican acercamiento o alejamiento de metas nacionales en relación con las de carácter internacional. El régimen de patrón oro tenía por objetivo sostener la convertibilidad monetaria a costa, si era necesario, de los objetivos nacionales. En contraste, al responsabilizar a los gobiernos del empleo y el crecimiento, el sistema de Bretton Woods invirtió los términos de la ecuación. En la actualidad, el péndulo monetario altera nuevamente su dirección, abandona el nacionalismo y vuelve al cosmopolitismo. En el caso de México — por fallas de adaptación-, este último cambio se tradujo en una lucha antiinflacionaria a ultranza que llevó al país a un cuasi estancamiento crónico, poniéndolo a la zaga de los procesos mundiales de desarrollo. 
I

\section{Introducción}

Las políticas nacionales han estado condicionadas en distinto grado por los paradigmas económicos, la visión dominante del Primer Mundo y la necesidad ineludible de ordenar la economía mundial en torno a un conjunto de reglas que han de respetarse a fin de hacer posible la convivencia entre naciones. Desde luego, todo orden internacional está sujeto a conflictos y asimetrías en su observancia; sin embargo, las ventajas de incorporarse a este orden o los inconvenientes de quedar fuera de él, resultan especialmente acentuados para los países periféricos.

Una parte esencial de los arreglos económicos entre naciones se expresa en regímenes monetarios y cambiarios que han de dar seguridad a las transacciones recíprocas. La abolición de las fronteras, el surgimiento de nuevas economías con peso universal, la re- volución tecnológica, han hecho indispensable el cambio y complicado enormemente el convenir acuerdos.

Desde la implantación del patrón oro, los regímenes monetarios han estado influidos por factores ideológicos que sirven precisamente para explicar, racionalizar e implantar la distribución de beneficios, costos y responsabilidades de cada orden económico mundial.

Este artículo pretende hacer un pequeño recuento de las oscilaciones históricas del péndulo monetario, destacando los movimientos que hacen acercarse o alejarse las metas puramente nacionales de aquellas del orden cosmopolita. Las consecuencias de esos movimientos tienen efectos significativos en el desarrollo y marcan la imbricación ineludible de los factores ideológicos en la vida económica de los países.

\section{II}

\section{Del patrón oro al sistema de Bretton Woods}

Aunque se oculten, son reconocibles los vuelcos del péndulo ideológico que rige la vieja oposición entre las exigencias del orden internacional y las demandas democráticas de cada país, nítidamente transparentes en las políticas monetarias. Hasta la primera guerra mundial, las ideologías dominantes, junto a la debilidad de los partidos de los trabajadores y las restricciones al voto ciudadano, hicieron prevalecer a las primeras sobre las segundas, hecho congruente con el impulso al comercio internacional dado por el Imperio Británico. El régimen de patrón oro tenía por objetivo central sostener de manera draconiana la convertibilidad monetaria, cargando los costos a los países deficitarios. ${ }^{1}$ Para estos efectos, los gobiernos y bancos centrales combatían los déficit de la balanza de pagos con medidas impopulares, impulsando la deflación de las economías mediante alzas en las tasas de interés y la reducción del circulante monetario, es decir, abatiendo desarrollo, importaciones y precios. $^{2}$

\footnotetext{
${ }^{1}$ Véanse Bloomfield (1959) y Eichengreen (1996a).
}

Después, las repercusiones devastadoras de la gran crisis de los años treinta, la universalización del sufragio y el fortalecimiento de partidos socialdemócratas, vienen a trastrocar los equilibrios políticos y los paradigmas económicos. Ganan las fuerzas ciudadanas para decidir soberanamente los derroteros nacionales, desaparece el colonialismo, se quiebra la espiral ascendente del intercambio internacional y las economías crecen como nunca. Las políticas keynesianas y la segunda guerra mundial sacan al mundo de la depresión y enderezan la lógica de las estrategias nacionales hasta hacer responsable al Estado del empleo pleno y del crecimiento de cada país.

Como resultado, se desmorona el sistema de cambios del patrón oro y se debilita el poder regulador de los bancos centrales; los países alzan barreras al comercio y a los flujos de capitales. En el orden internacional

\footnotetext{
${ }^{2}$ Las paridades fijas, aparte de constituir un compromiso gubernamental de impedir o limitar las fluctuaciones cambiarias, sirven de ancla nominal a las operaciones y expectativas de los agentes productivos sobre el comportamiento de la política monetaria.
} 
se finiquitan los acuerdos y se crean las llamadas instituciones de Bretton Woods. Surge un nuevo régimen monetario-cambiario que, a diferencia del anterior, no estipula tipos de cambio fijos, sino revisables, siempre y cuando se experimente un desequilibrio fundamental invocado por el país afectado y aceptado por el Fondo Monetario Internacional (FMI). El propio Fondo concedía algún financiamiento de la balanza de pagos y permitía controles nacionales a los movimientos de capitales y en la práctica también al intercambio de bienes y servicios.

El sistema surgido de Bretton Woods reflejó los cambios políticos que tornaron inviable la deflación de las naciones deficitarias como única vía de corregir los desajustes de pagos y que abrieron la puerta al período de más intenso desarrollo de la economía mundial, sobre todo de las zonas periféricas. La revisión de las paridades servía para eliminar los déficit externos con menores costos nacionales. A su vez, los controles permitían eludir los efectos de los movimientos bruscos o especulativos de capitales y frenar la acumulación de saldos comerciales deudores.

Sin embargo, el convenio sólo admitía alteraciones de la paridad asociadas a desequilibrios graves, reconocidos por el Fondo Monetario Internacional, reduciendo así en los hechos la flexibilidad cambiaria. Antes de reconocer el imperativo de una devaluación, antes de admitir el fracaso de la política monetaria, los gobiernos y bancos centrales se esforzaban por mantener sin variación el tipo de cambio, reviviendo la oposición entre el ajuste externo y los objetivos de desarrollo nacionales. Con tal fin se disponía de instrumentos para intensificar el proteccionismo (aranceles, permisos de importación, etc.) y corregir al menos temporalmente los desajustes de pagos.

De otro lado, por presión estadounidense, el sistema de Bretton Woods buscó el restablecimiento de la convertibilidad monetaria, como requisito previo al florecimiento del comercio internacional. Fracasan los intentos de hacerlo con la libra esterlina (1947), mediante la Carta de La Habana, que trató de crear una organización internacional del comercio; también fueron escasos los primeros avances del Acuerdo General de Aranceles Aduaneros y Comercio (GATT), mientras la creación de la Unión Europea de Pagos (19501958) tuvo un sesgo regional más que multilateral.

Esos ensayos anunciaban, sin embargo, el inicio del cambio de dirección del péndulo, que abandonaba el nacionalismo y volvía al cosmopolitismo. Al comienzo, los intentos de reimplantar la convertibilidad monetaria universal enfrentaron conflictos de interés entre los países desarrollados. Las naciones europeas, devastadas por la guerra, resistían la liberalización del comercio; para nivelar sus balanzas de pagos necesitarían devaluaciones cambiarias sustantivas que deteriorarían los estándares de vida de la población, sin resolver a fondo las limitaciones de su capacidad productiva y exportadora (en 1947 Europa registró un déficit conjunto de 7.500 millones de dólares). ${ }^{3}$ Por su lado, los Estados Unidos consideraban indispensable alcanzar la convertibilidad para que sus exportaciones encontrasen condiciones equitativas de competencia. A fin de facilitar la solución del problema, el gobierno de ese país aceptó incrementar sustancialmente el financiamiento a Europa a través del Plan Marshall y otros expedientes.

Entre 1959 y 1961, los países europeos restablecieron la convertibilidad de la cuenta corriente, pero dejaron vigentes los controles en la cuenta de capital. El péndulo de la política monetaria siguió avanzando, apartándose del nacionalismo económico, al hacerse más y más convergentes los intereses de los miembros del mundo desarrollado, aunque no desaparecieran todas las dificultades y surgieran nuevos problemas.

A partir de ahí, el saneamiento de los desequilibrios de pagos de Europa y Japón, y el ascenso del comercio, de los flujos de inversión y de las operaciones de empresas internacionales, multiplican en número y fuerza a los actores privados en el escenario mundial, mientras se empequeñece correlativamente la influencia de los gobiernos nacionales. Los países comienzan a restablecer la convertibilidad de las transacciones en cuenta corriente de la balanza de pagos, haciendo cada vez más difícil el ejercicio de controles efectivos en la cuenta de capital, hasta que estos últimos son desmantelados en la mayoría de los países.

Desde la década de 1960 Europa y Japón crecían y hacían acrecentar su comercio, tornándose atractivos para la inversión extranjera, en tanto que los Estados Unidos comenzaron a registrar desbalances comerciales persistentes. ${ }^{4}$ A la vez y paradójicamente, el dólar consolidaba su posición como moneda de reserva, mientras se acrecentaban los desajustes estadounidenses de balanza de pagos, creando el peligro de que ese

\footnotetext{
${ }^{3}$ En 1949, los países europeos devaluaron alrededor del $30 \%$ sus monedas y, aun así, no pudieron suprimir los controles a las importaciones, pero sí abrieron el camino al saneamiento de sus balanzas de pagos (véase Eichengreen, 1996a, p. 98).

4 Ya en 1960, la balanza de pagos de los Estados Unidos mostró debilidades que se acentuaron considerablemente con el gasto de la guerra de Vietnam y los esquemas simultáneos de la Gran Sociedad.
} 
país renunciase a sostener la convertibilidad oro-dólar, provocando así serios problemas a la liquidez en el mundo (el dilema de Triffin $)^{5}$ y el desplome consiguiente del sistema cambiario de Bretton Woods.

\section{III}

\section{Después de Bretton Woods}

El sistema monetario de la posguerra, que descansaba en el compromiso de Estados Unidos para sostener el precio fijo del oro, se erosionó no sólo por el riesgo nacido de déficit estadounidenses acumulativos, sino por el hecho de que las reservas de los bancos centrales estaban integradas mayoritariamente por dólares. En 1971, ante el éxodo de sus reservas y el rechazo a la solución alternativa de deflacionar su economía, Estados Unidos cancela el compromiso de entregar oro ilimitadamente al precio de 35 dólares la onza, derrumbando el régimen cambiario de Bretton Woods. Desde entonces se ensayan variados regímenes cambiarios que paulatinamente se decantan en los extremos, sea en paridades flotantes más o menos libres o en paridades fijas, ${ }^{6}$ no sin crear serias desalineaciones cambiarias entre países, movimientos especulativos, volatilidad y contagios que están lejos de haberse corregido.

Esos acontecimientos devuelven el meollo del anclaje del sistema monetario mundial a la responsabilidad de las autoridades nacionales, supervisadas férreamente por los organismos multilaterales (Banco Mundial, Fondo Monetario Internacional, Organización Mundial del Comercio). Hacer que los gobiernos asuman papeles análogos a los que tenían con el patrón oro implica costos, abrazar políticas y vencer resistencias que violentan la voluntad popular en la toma de decisiones en aras de atender normas y exigencias del orden internacional. A mayor abundamiento, la observancia de las reglas internacionales reconoce

\footnotetext{
5 Véase Triffin (1960).

${ }^{6}$ La diversidad de sistemas va desde la dolarización y las juntas monetarias, tipos de cambio fijos con una determinada moneda o con canastas de monedas, bandas fijas o ajustables, y paridades deslizantes, hasta la flotación administrada y la flotación libre, entre otros. Al respecto véase Mussa y otros (2000); Cartens y Werner (1999); Ibarra y Moreno-Brid (2001). Los regímenes de tipo de cambio fijo poco a poco se reducen en número. Por lo general lo adoptan economías pequeñas, en proceso de integración - como las europeas - o las que han elegido el camino de la dolarización. Véanse Eichengreen y Freden (1998); Obstfeld (1997); Ibarra y Moreno-Brid (2001); Hauke y Schuler (1993).
}

asimetrías: son inflexibles con el mundo en desarro1lo, incluso con los países emergentes, y laxas con la naciones desarrolladas, como lo demuestra la magnitud de los desequilibrios estadounidenses de pagos externos o los déficit gubernamentales que ya se sitúan entre el 4\% y el 8\% del PIB en Europa, Japón y Estados Unidos. $^{7}$

En las últimas tres décadas se vive una innegable paradoja. Mientras los países pueden asumir teóricamente el régimen cambiario de su elección -incluso el que otorgue la más plena autonomía a sus políticas internas-, el orden internacional exige sostener la apertura con estabilidad de precios de cada país a fin de eliminar riesgos a los flujos financieros y de comercio, variables en las cuales se concentran los grandes intereses hegemónicos transnacionales.

En consonancia con los hechos enunciados, los paradigmas ideológicos se han movido de hacer el elogio del desarrollo y del empleo a tomar el combate a la inflación, como el objetivo social por excelencia. En esa lógica, los gobiernos nacionales y sus instituciones se tornan sospechosos de procurar ventajas políticas a costa de los equilibrios macroeconómicos fundamentales. Hay desconfianza ideológica en el comportamiento del Estado y confianza plena en los mercados. Por eso, frecuentemente se inculpa a la política fiscal de inducir el gasto por encima de la tasa natural de crecimiento o empleo. Y se abraza el criterio del equilibrio presupuestario en cualquier circunstancia, es decir, se renuncia implícitamente a la instrumentación de medidas fiscales desarrollistas o contracíclicas.

En el ámbito de la política monetaria, los cambios de enfoque abarcan no sólo al diseño de las políticas, sino el de las instituciones. La visión posmoderna de

\footnotetext{
${ }^{7}$ Los déficit fiscales de los países emergentes y en desarrollo han alcanzado en promedio el 3\% del producto (México 1,5\%), mientras los de las naciones avanzadas ascendieron al 4\% (Estados Unidos $5,9 \%$, Japón $8,2 \%$, Alemania 4,0\%, Francia 4,1\%). Los datos provienen de FMI (2004).
} 
la banca central destaca la necesidad de disolver los problemas de la llamada "inconsistencia temporal", esto es, las incongruencias gubernamentales entre anunciar políticas de combate a la inflación y luego contradecirlas en función de ganancias políticas o electorales que la teoría económica usualmente presupone transitorias. El descrédito real o imaginado de los gobiernos lleva a declarar la independencia de los bancos centrales para que persigan sin contaminación política objetivos de estabilización de precios. ${ }^{8}$ La credibilidad externa se torna predominante.

Aun así, la menor credibilidad intrínseca de los bancos centrales en regímenes de flotación requiere el anclaje complementario de la política fiscal consistente en el compromiso de reducir los déficit presupuestarios y obligar a que toda expansión del gasto tenga que financiarse con impuestos, o mediante operaciones de mercado abierto (impuestos diferidos en el tiempo) que equilibren su impacto monetario. Cuentas fiscales sin déficit, bancos centrales autónomos y la supresión de las políticas industriales conforman la más impresionante cesión de soberanía económica de los países latinoamericanos, que algunos llevan o llevarían hasta la supresión de las monedas nacionales, esto es, la dolarización —o la creación de las llamadas juntas monetarias-, aun sin mediar las instituciones y los acuerdos de respaldo mutuo y de participación en el diseño de las políticas económicas conjuntas, como las de que tiene la Unión Europea. De proseguir esos procesos, se llegaría a formar uniones monetarias o uniones económicas en las cuales los costos estarían asimétricamente cargados no al país dominante, sino a su periferia.

En suma, el anclaje simultáneo fiscal y monetario constituye una postura destinada a ganar credibilidad externa en torno a las políticas antiinflacionarias de los gobiernos, que reduce en alto grado los márgenes de maniobra de las políticas públicas internas y les imprime un carácter marcadamente procíclico y poco democrático. Es decir, hay aquí un intento por supri-

$8 \mathrm{Si}$ bien los gobiernos pueden dar sorpresas monetarioinflacionarias, los intentos resultarán fallidos — reza la teoría- si los agentes económicos los anticipan, dando como resultado más inflación, sin efectos positivos sobre el desarrollo (véanse Barro y Gordon, 1983, pp.101-121). Enfoques más generales o detallados se encuentran en Rogoff (1985, pp. 1169-1190); Giavazzi y Pagano (1988, pp. 1055-1082); Bernhard y otros (2002, pp. 693-723). mir las diferencias de precios y de tasas de interés entre países por la vía de la deflación hasta igualar esas variables con las de los países líderes, pasando por alto los distintos arreglos institucionales y de fuerzas políticas de cada nación.

El regreso del péndulo monetario guarda, sin embargo, una diferencia con el viejo régimen de patrón oro. Antes, las políticas recesivas se instrumentaban pari passu con el surgimiento de los desequilibrios; hoy se implantan preventivamente, sometiendo a muchos países periféricos a una suerte de cuasi estancamiento crónico que les impide resolver las fallas estructurales de los desequilibrios de pagos. El dilema monetario se reduce entonces a la oposición entre credibilidad externa y escrutinio democrático interno sobre las políticas públicas.

Los énfasis de los paradigmas económicos en materia cambiaria se han desplazado con las nuevas realidades. En el decenio de 1990 dejó de discutirse el problema de las crisis, el modo de evitar altos y crecientes desajustes de la cuenta corriente y de flujos de capital insostenibles, o planteamientos sobre la flexibilidad comparativa de precios, salarios y tasas de interés, siempre relacionados con objetivos nacionales de crecimiento y empleo. Hoy el foco del análisis de la política anticrisis queda centrado en cómo sostener y acrecentar los flujos internacionales de capital, teniendo como meta de trasfondo la estabilización de precios de las economías. Por eso, se ha pasado de buscar márgenes de maniobra desarrollistas para la política monetaria, a ceder casi por entero su independencia frente a las exigencias de la globalización. ${ }^{9}$

\footnotetext{
${ }^{9}$ Según Summers, los requisitos previos para reducir los riesgos de crisis financieras y cambiarias tienen que ver con la cuenta de capitales de la balanza de pagos, mientras no hace mención de los problemas de la cuenta corriente, que se consideran subordinados a la primera. En esa lógica, la inversión extranjera y los créditos alcanzan un papel protagónico para el mundo en desarrollo, pese a la volatilidad de sus flujos. Esos requisitos son los siguientes: i) mantener o crear un sistema financiero fuerte (bancos bien capitalizados y supervisados, códigos efectivos de gobierno corporativo, estado de derecho, protección de los contratos) que haga sostenible acumular deudas sustantivas; ii) adoptar un régimen cambiario de tasa fija o flotación libre que facilite el acceso a los mercados de capitales; iii) tener un clima macroeconómico estable, que minimice los riesgos monetarios y fiscales; iv) acumular reservas en proporción adecuada a los pasivos del país (véase Summers, 2000, pp. 1-16.)
} 


\section{IV}

\section{La respuesta mexicana}

México no es una excepción en la materia. La autonomía concedida al Banco de México por ley de 1993 le fija el objetivo único o principalísimo de combatir la inflación, al tiempo que limita la concesión de crédito al gobierno. Como en otros casos, es ambiguo si la independencia institucional abarca sólo el manejo del instrumental monetario o incluye la capacidad de fijar unilateralmente metas de alcance nacional que pudiesen ser distintas o hasta contraponerse a las del propio gobierno y los órganos legislativos.

Sin duda, nuestra historia reciente y lejana registra casos en que los gobiernos usaron y abusaron de la política monetaria y cambiaria para evadir los sacrificios políticos de ajustar a tiempo la economía cuando resultaba indispensable. Pero también la historia del Banco de México y de sus fideicomisos recogen esfuerzos exitosos: al canalizar fondos privados y públicos para fortalecer la capacidad productiva - junto a la banca de desarrollo-, hicieron posible la etapa de más intenso crecimiento del país, la de 1940$1980 .{ }^{10}$

La liberalización de la cuenta de capital abrió el país a la volatilidad, sobre todo de los flujos de capital de corto plazo. ${ }^{11}$ Los acusados movimientos en las cotizaciones de las principales monedas internacionales (euro, dólar, yen), así como los contagios financieros recientes, demuestran que los países en desarrollo difícilmente pueden guarecerse de los movimientos financieros desestabilizadores cuando surgen en una economía globalizada.

Más aún, en nuestro caso se combatió la inflación con tasas de interés elevadas en términos internacionales, creando incentivos para que los inversionistas foráneos trajesen fondos de corto plazo que se mante-

\footnotetext{
${ }^{10}$ Aparte de los cajones crediticios favorecedores de actividades calificadas de prioritarias, destacan los Fideicomisos Instituidos en Relación con la Agricultura (FIRA), el Fondo de Fomento de las Exportaciones de Productos Manufacturados, el Fondo de Equipamiento Industrial (FONEI), el Fondo de Operación y Financiamiento Bancario de la Vivienda (FOVI), el Fondo Nacional de Fomento al Turismo (Fonatur) y el Fondo para el Desarrollo Comercial (FIDEC).

${ }^{11}$ Las ventajas o inconvenientes de abrir la cuenta de capitales han sido objeto de un intenso debate internacional; entre los principales críticos destacan Tobin, Bhagwati y Rodrik. Véanse Ul Haq, Kaul y Grunberg (1996), Bhagwati (1998) y Rodrik (1998).
}

nían en el país mientras no se elevasen las expectativas de devaluación. La sobrevaluación resultante del tipo de cambio sólo se puede combatir con operaciones costosas de esterilización y acumulación de reservas; y también lleva el riesgo inherente de la cesación de los flujos de entrada y aun de salidas precipitadas de capitales, como las experimentadas entre 1994 y 1995. Esas tendencias a la sobrevaluación se vieron acentuadas en el decenio de 1990 por los intensos procesos de extranjerización de un buen número de empresas públicas y privadas, los cuales determinaron fuertes entradas de divisas que no se invirtieron en acrecentar significativamente las capacidades productivas y exportadoras.

Como consecuencia, el proceso de ajuste deflacionario ha sido exitoso en acercar la inflación nacional a la estadounidense. Los costos históricos han sido el de la sobrevaluación cambiaria con efectos destructivos en la competitividad de los productores nacionales y el de caminar en sentido opuesto a la estrategia de crecimiento hacia fuera, desaprovechando la demanda de los mercados internacionales.

El abandono del régimen de paridad fija obedece, en consecuencia, a dos factores centrales que quizás poco tengan que ver con metas desarrollistas propiamente tales. México tradicionalmente usó tipos de cambio fijos asociados al dólar, como anclaje monetario y, en general, de todas las políticas públicas. La importancia de esa función no fue trivial: aportaba certeza en un país regido por un gobierno semiautoritario, de partido hegemónico, cuyas decisiones y acciones resultaban frecuentemente opacas al exterior, a los agentes económicos y a los ciudadanos. Hasta finales del decenio de 1980, el informe anual del Presidente al Congreso de la Unión era casi el único documento que, a la par de abundar en lo hecho por el gobierno, señalaba los nuevos derroteros económicos y políticos. Ahí se anunciaba el monto de las reservas internacionales y se atenuaban las asimetrías de información entre gobierno, de un lado, y partidos políticos y ciudadanos, del otro. Sin embargo, las aperturas comercial y financiera dejaron inerme a la economía nacional frente a los flujos irrestrictos, no siempre estabilizadores, de capitales y mercaderías, así como 
al contagio de crisis externas. ${ }^{12}$ Por otro lado, desde el ángulo de la política nacional, resultaba y resulta deseable evitar las crisis devaluatorias sexenales debidas esencialmente a la acumulación de diferencias de precios con el exterior, que se traducían en déficit comerciales o endeudamientos imposibles de financiar. La flotación crea una vía de escape a ambos problemas, pero exige mayor rigor monetario y fiscal, y mayores sacrificios de crecimiento, a fin de reducir las presiones de precios, sean ellas reales o de credibilidad, y acercar la inflación nacional a la estadounidense.

Tampoco el sistema de flotación resuelve todos los posibles excesos de las políticas públicas, aunque bien puede cambiar su dirección. Hoy los incentivos se han invertido: la tentación de los bancos centrales independientes no reside tanto en facilitar expansiones económicas sostenibles o insostenibles, sino en elevar más de la cuenta las tasas de interés o permitir la sobrevaluación de la moneda a fin de obtener ganancias costosas y temporales en abatir la inflación, su principal o única responsabilidad. El menor crecimiento resultante acota los incentivos para invertir, innovar y mejorar la competitividad, mientras los costos mayores, artificiales, a los productores nacionales, facilitan su desplazamiento por oferentes del exterior, ${ }^{13}$ acrecentando la dependencia de las importacio- nes. Así se cierra el círculo del estancamiento estabilizador al empobrecerse repetitivamente los alicientes al desarrollo y deteriorarse la posición de la concurrencia nacional en los mercados foráneos.

No obstante alguna corrección cambiaria reciente, el peso ha estado casi sistemáticamente sobrevaluado entre episodios de crisis devaluatorias. Si bien ese proceder contribuye a reducir temporalmente las presiones inflacionarias, daña a los productores nacionales. Aquí reside una de las razones que explican la pérdida de mercados externos y que los déficit comerciales fluctúen alrededor de los 9.000 millones de dólares, mientras el conjunto de América Latina tiene superávit (27.000 millones en 2003). ${ }^{14}$ En contraste, China, Japón, la República de Corea y la provincia de Taiwán mantienen deliberadamente subvaluados sus signos monetarios en apoyo a su comercio exportador, mediante intervenciones directas en los mercados cambiarios, aun contraviniendo las reglas del FMI. ${ }^{15}$

La política fiscal completa el anclaje antiinflacionario del manejo monetario al comprimir deliberadamente los déficit fiscales por debajo de la media de los países en desarrollo ${ }^{16} \mathrm{y}$ al reducir el gasto público automáticamente, por ley, cuando caen los ingresos del gobierno, independientemente de la posición de la economía en el ciclo económico.

\section{$\mathrm{V}$}

\section{A modo de conclusión}

En suma, se ha caído en un vacío estratégico que lleva al país a un cuasi estancamiento permanente o a seguir a la zaga internacional de los procesos de desarrollo. Lo viejo no funciona y lo nuevo no se sabe manejar. De un lado, la apertura, la intensificación del cambio tecnológico mundial, las hegemonías económicas de nuevo cuño, han hecho anacrónicas e inviables las estrategias proteccionistas. De otro lado, la obsesión antiinflacionaria del cosmopolitismo de la globalización se traduce en nuestro medio en políticas que derrotan a la estrategia de crecimiento hacia afuera. El

\footnotetext{
12 Véanse Garber y Svensson (1995, pp. 1865-1912); Obstfeld y Rogoff (2002, pp. 503-535); Bergsten y Williamson (2003), y Eichengreen (1996b).

13 Véase Ibarra (1999, pp. 139-160, y 2001, pp. 259-280). También Villarreal (2003).
}

uso de altas tasas de interés, de la sobrevaluación cambiaria y de presupuestos equilibrados (no sólo en la cuenta corriente, sino incluida la de capital), como armas estabilizadoras, sitúan a los productores nacionales directa e indirectamente frente a la tarea hercúlea no sólo de compensar rezagos históricos frente a los mejores productores del mundo, sino de hacer otro tanto con factores artificiales de una política económica que les restan competitividad.

\footnotetext{
14 Las cifras son de la CEPAL (2003).

${ }^{15} \mathrm{La}$ acumulación de reservas de esos cuatro países ya asciende a más de 1,7 billones españoles (trillones anglosajones) de dólares, prueba manifiesta de las enormes desalineaciones cambiarias del mundo y de la necesidad de reconstruir la arquitectura monetaria del orden mundial (véase Bergsten, 2004).

16 Véanse las cifras de la nota 7.
} 
El vacío estratégico tiene efectos no sólo en el período de deflación — cuando se intenta bajar el alza de precios a los estándares internacionales-, sino que se extiende en el tiempo. Los rezagos resultantes en la formación de capital humano, en la inversión física, la modernización tecnológica, la inserción en las redes transnacionales de producción y comercio, frecuentemente se tornan irrecuperables o difíciles de recuperar durante largos años. Ganar el equilibrio en las metas sociales y en su reflejo de las políticas públicas es, sin duda, la tarea vertebral y pendiente de la política nacional.

\section{Bibliografía}

Barro, R. y D. Gordon (1983): Rules, discretion and reputation in a model of monetary policy, Journal of Monetary Economics, vol. 12, $\mathrm{N}^{\circ} 1$, Rochester, University of Rochester.

Bergsten, R. (2004): Testimony before the Committee on Banking, Housing and Urban Affairs, Washington, D.C., Institute for International Economics.

Bergsten, R. y J. Williamson (2003): Dollar Overvaluation and the World Economy, Washington, D.C., Institute for International Economics.

Bernhard, W. y otros (2002): The political economy of monetary institutions, International Organization, vol. 56, $\mathrm{N}^{\mathrm{o}} 4$, Cambridge, Massachusetts, The MIT Press.

Bhagwati, J. (1998): Why Free Capital Mobility May be Hazardous Health: Lessons from the Latest Financial Crises, Cambridge, Massachusetts, National Bureau of Economic Research.

Bloomfield, A. (1959): Monetary Policy under the International Gold Standard, Nueva York, Federal Reserve Bank of New York.

Cartens, A. y A. Werner (1999): Mexico's Monetary Policy Framework, documento de investigación, No 9005, México, D.F., Banco de México.

CEPAL (Comisión Económica para América Latina y el Caribe) (2003): Balance preliminar de las economías de América Latina y el Caribe, 2003, LC/G.2223-P, Santiago de Chile. Publicación de las Naciones Unidas, $\mathrm{N}^{\circ}$ de venta: S.03.II.G.186.

Eichengreen, B. (1996a): Globalizing Capital: A History of the International Monetary System, Nueva Jersey, Princeton University Press.

(1996b): Speculative Attacks on Pegged Exchange Rates, California, University of California Press.

Eichengreen, B. y S. Freden (1998): Forging an Integrated Europe, Ann Arbor, Michigan, University of Chicago Press.

FMI (Fondo Monetario Internacional) (2004): World Economic Outlook, Washington, D.C.

Garber, P. y N. Svensson (1995): The operation and collapse of fixed exchange regimes, en G. Grossman y K. Rogoff (comps.), Handbook of International Economics, vol. 3, Amsterdam, North-Holland.

Giavazzi, F. y M. Pagano (1988): The advantage of tying one's hand: EMS discipline and Central Bank credibility, European Economic Review, vol. 32, № 5, Amsterdam, Elsevier.
Hauke, S. y K. Schuler (1993): Currency Boards and Their Relevance for Latin America, World Bank Discussion Paper, $\mathrm{N}^{\circ} 27$, Washington, D.C., Banco Mundial.

Ibarra, D. (1999): ¿Es aconsejable una política industrial en México?, Política y economía, México, D.F., Miguel Ángel Porrúa. (2001): Política cambiaria, paridad cambiaria y globalización, Testimonios críticos, México, D.F., Cántaro Editores.

Ibarra, D. y J.C. Moreno-Brid (2001): Currency boards and monetary unions: the road ahead or cul de sac for Mexico's exchange rate policy?, en M. Puchet y L. Punzo (comps.), Mexico Beyond Nafta: Perspectives for the European Debate, Londres, Routledge.

Mussa, M. y otros (2000): Exchange Rate Regimes in an Increasingly Integrated World Economy, Occasional Paper, $\mathrm{N}^{\circ} 193$, Washington, D.C., Fondo Monetario Internacional.

Obstfeld, M. (1997): Europe's gamble, Brookings Papers on Economic Activity, $\mathrm{N}^{\circ}$ 2, Washington, D.C., Brookings Institution Press.

Obstfeld, M. y K. Rogoff (2002): Global implications of self-oriented national monetary rules, Quarterly Journal of Economics, vol. $117, N^{\circ} 2$, Cambridge Massachusetts, The MIT Press.

Rodrik, D. (1998): Who needs capital account convertibility?, en P. Kenen (comp.), Should The IMF Pursue Capital Account Convertibility?, Princeton Studies in International Finance, $\mathrm{N}^{\mathrm{o}}$ 207, Princeton University, Princeton.

Rogoff, K. (1985): The optimal degree of commitment to an intermediate monetary target, The Quarterly Journal of Economics, vol. 100, № 4, Cambridge, Massachusetts, The MIT Press.

Summers, L. (2000): International financial crises: causes, prevention, and cures, American Economic Review, vol. 90, $\mathrm{N}^{\circ} 2$, Nashville, Tennessee, American Economic Association, mayo.

Triffin, R. (1960): Gold and the Dollar Crisis: The Future of Convertibility, New Haven, Connecticut, Yale University Press.

Ul Haq, M., I. Kaul e I. Grunberg (comps.) (1996): The Tobin Tax: Coping with Financial Volatility, Nueva York, Oxford University Press.

Villarreal, R. (2003): El reto de Fox: del estancamiento estabilizador a la reactivación y crecimiento competitivo con estabilidad, Revista del Instituto Mexicano de Ejecutivos de Finanzas, México, D.F., Instituto Mexicano de Ejecutivos de Finanzas, agosto. 